\title{
RESOLVING CYCLIC AMBIGUITIES AND INCREASING ACCURACY AND RESOLUTION IN DOA ESTIMATION USING ARRAY ROTATION
}

\author{
AbdelhamidDjouadi ${ }^{1}$ and NebojsaI. Jaksic ${ }^{2}$ \\ ${ }^{1}$ Nokia, 4575 Rings Rd, Dublin, Ohio, USA \\ ${ }^{2}$ Colorado State University - Pueblo, 2200 Bonforte Blvd., \\ Pueblo, Colorado, USA
}

\begin{abstract}
A method to resolve cyclic ambiguities and increase the accuracy and the resolution in the direction-of-arrival (DOA) estimation using the Estimation of Signal Parameters via Rotational Invariance Technique (ESPRIT)algorithm is proposed. It is based on rotating the array and sampling the received signal at multiple positions. Using this approach, the gain in accuracy and resolution is addressed as function of the mean and variance of the DOA. Simulations results are provided as a means of verifying this analysis.
\end{abstract}

\section{KEYWORDS}

Direction Of Arrival (DOA) estimation, Estimation of Signal Parameters via Rotational Invariance Technique (ESPRIT), Total Least Square (TLS), Cyclic Ambiguities

\section{INTRODUCTION}

Because of the widespread use of sensor arrays, and the continuing development of their capabilities, sensor arrays have experienced an increased range of applications. One of these applications that has been given special attention is the high resolution direction-of-arrival (DOA) estimation. This estimation is mainly based on the processing of the received signal to extract the desired parameters of DOA of plane waves upon which the sensor outputs depend. The estimation of Signal Parameters by Rotational Invariance Techniques (ESPRIT) is one of the many approaches that have been used for implementing the DOA functions[1].In essence, and for the narrowband direction-of-arrival case, ESPRIT algorithm estimates a unitary diagonal matrix $\Phi$ with diagonal elements, given by $\varphi_{i}=e^{-j \mu_{i}} ; i=1, \ldots d$, where $d$ is the number of sources impinging on the array from distinct locations $\theta_{1}, \theta_{2}, \ldots, \theta_{d}$, as shown in Figure 1 , and where the parameters $\mu_{i} ; i=1, \ldots, d$, denote the phase shifts to be estimated. For a uniform linear array (ULA), it is well known that the phase shift $\mu$ is related to the angle of arrival $\theta$ by

Jae-Kwang Lee et al. (Eds) : CCSEA, AIFU, DKMP, CLOUD, EMSA, SEA, SIPRO - 2017

pp. 121-138, 2017. (C) CS \& IT-CSCP 2017

DOI : $10.5121 /$ csit.2017.70212 


$$
\mu=2 \pi D \sin \theta / \lambda
$$

where $\lambda$ is the wavelength of the narrow-band signal and $D$ is the displacement vector between two sub-arrays.

Various algorithms were proposed successively to improve the ESPRIT performance. However, much of the studies show that the performance of these algorithms depends only on the number of snapshots $(n)$, the number of array sensors $(m)$, and the signal-to-noise $(S N R)$ of the received array signals [2]-[5].

Further in [6]-[8], it was shown that the performance is improved by using multi-scale method, where the short $(D \leq \lambda / 2)$ and long baselines $(D>\lambda / 2)$ are, respectively, utilized to derive the coarse unambiguous and fine ambiguous estimates for each signal. The final DOA estimate in this case is determined by using the coarse estimate to disambiguate the fine estimate.

This work shows that the performance of the ESPRIT algorithm is also constrained by the angle of arrivals of incidence with which the sources are impinging the array, and that the ESPRIT algorithm produces ambiguities in the estimated DOAs when the antenna element spacing on the linear array has a measurement error [9]-[10], or when the sources are impinging on the array with an angle of incidence along-side the ULA, especially for low SNR and limited number of snapshots.

The purpose of this work is to show that by rotating the array and sampling the received signal at multiple positions, ambiguities in the DOA estimates are resolved and also more accurate DOA estimates are obtained. Using this approach, the gain in accuracy and resolution are addressed as

function of the mean and variance of the angle of arrival errors of the sources. An analysis of simulation results is provided to verify the method described.

\section{PROBLEM FORMULATION}

This section formulates the DOA estimation problem when using the ESPRIT algorithm and shows that its performance depends on the angle-of-arrival (incidence) with which the source is impinging the array. Also it describes the ambiguities produced in the estimated DOA results, when the antenna element spacing on a linear array is more than half a wavelength because of measurement errors, or when the sources are impinging on the array at an angle of incidence along-side the ULA.

\subsection{Data Model}

Consider an array consisting of two sub-arrays as shown inFigure1. Each sub-array consists of $m$ elements. The two sub-arrays are assumed to be overlapping, and separated by a displacement vector $D$ (where $D$ is a fixed distance equal or smaller than half a wavelength). Assume that $d<m$ narrow-band sources impinge on the array from distinct locations $\theta_{1}, \theta_{2}, \ldots, \theta_{d}$. For simplicity, it is assumed that the sources are narrow-band, no-coherent, coplanar, and that they are in the far field of the array. This assumption allows modeling the propagation delays between sensor elements as simple phase shifts, and thus the only parameters that characterize the sources 
locations are their DOAs. In this case, the output at any sensor of the array is the superposition of the individual emitter's signals- $s_{1}(t), s_{2}(t), \ldots, s_{d}(t)$, weighted by the sensor response.

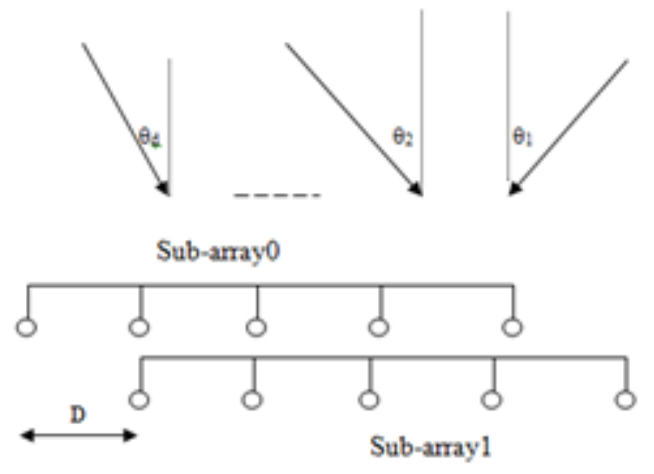

Figure 1. Array Configuration

The received signal at the $k^{\text {th }}$ sensor can be written as

$$
x_{k}(t)=\sum_{i=1}^{d} a_{k}\left(\theta_{i}\right) s_{i}(t) e^{-j \omega_{0} \tau_{k}\left(\theta_{i}\right)}+n_{k}(t)
$$

where $\tau_{k}\left(\theta_{i}\right)$ is the propagation delay between a reference point and the $k^{\text {th }}$ sensor for the $i^{\text {th }}$ waveform impinging on the array from direction $\theta_{i}, a_{k}\left(\theta_{i}\right)$ is the corresponding sensor element complex response (gain and phase) at frequency $\omega_{0}$, and $n_{k}(t)$ is the additive noise at the $k^{\text {th }}$ sensor. If we let $\underline{a}\left(\theta_{i}\right)=\left(a_{1}\left(\theta_{i}\right) e^{-j \omega_{0} \tau_{1}\left(\theta_{i}\right)}, a_{2}\left(\theta_{i}\right) e^{-j \omega_{0} \tau_{2}\left(\theta_{i}\right)}, \ldots, a_{m}\left(\theta_{i}\right) e^{-j \omega_{0} \tau_{m}\left(\theta_{i}\right)}\right)^{T}$ to be the data model representing the outputs of the $m$ sensors of the first (reference) sub-array, then the data model representing the outputs of the $m$ sensors is given by

$$
X_{0}(t)=\sum_{i=1}^{d} \underline{a}\left(\theta_{i}\right) s_{i}(t)
$$

Now by letting $A(\theta)=\left(\underline{a}\left(\theta_{1}\right), \underline{a}\left(\theta_{2}\right), \ldots, \underline{a}\left(\theta_{d}\right)\right), S(t)=\left(s_{1}(t), s_{2}(t), \ldots ., s_{d}(t)\right)^{T}$, and $N_{0}(t)=\left(n_{01}(t), n_{02}(t), \ldots, n_{0 m}(t)\right)^{T}, X_{0}(t)$ can be rewritten as:

$$
X_{0}(t)=A(\theta) S(t)+N_{0}(t)
$$

where $A(\theta)$ is called the direction matrix. The columns of $A(\theta)$ are elements of a set, termed the array manifold, composed of all array response vectors obtained as $\theta$ ranges over the entire space. 
Likewise, the data model representing the outputs of the $m$ sensors of the second sub-array is given by

$$
X_{1}(t)=A(\theta) \Phi S(t)+N_{1}(t)
$$

where $N_{1}(t)=\left(n_{11}(t), n_{12}(t), \ldots, n_{1 m}(t)\right)^{T}$, and where $\Phi$ is the previously defined unitary diagonal matrices with diagonal elements $\varphi_{i}$ given by $\varphi_{i}=\exp \left\{-j 2 \pi D \sin \theta_{i} / \lambda\right\}$, and where $\lambda$ is the wavelength of the narrow-band signal. $N_{0}(t)$ and $N_{1}(t)$ represent the uncorrelated noise present at each antenna element of the first and second sub-arrays, respectively.

In order to estimate the DOA, ESPRIT exploits the shift structure inherent in the relevant signal subspace that contains the output $Z(t)$ given by:

$$
Z(t)=\left(\begin{array}{l}
X_{0}(t) \\
X_{1}(t)
\end{array}\right)=\bar{A} S(t)+\left(\begin{array}{c}
N_{0}(t) \\
N_{1}(t)
\end{array}\right)
$$

where $\bar{A}=\left(\begin{array}{l}A(\theta) \\ A(\theta) \Phi\end{array}\right)$

The DOA's estimation is achieved by separating the $2 m$-dimensional complex vector space $C^{2 m}$ of output snapshots into orthogonal subspaces, namely the signal subspace and the noise subspace. This is achieved by performing the eigen decomposition of the covariance matrix

$$
R_{Z Z}=E\left(Z^{*}(t) Z(t)\right)=\bar{A} R_{s S} \bar{A}^{*}+\sigma^{2} I
$$

where $E\left(\right.$.) denotes expectation, $R_{z z}$ is the covariance matrix of measurements, $R_{s s}$ is the positive definite of the stationary (zero-mean) of the signals, and $\sigma^{2} I$ is the spatial correlation matrix of the uncorrelated noise vector $N(t)=\left(N_{0}(t), N_{1}(t)\right)^{T}$. In practice, the covariance matrix is obtained by first collecting $n$ snapshots, $Z\left(t_{1}\right), Z\left(t_{2}\right), \ldots, Z\left(t_{N}\right)$ of the output, and then computing the sample covariance matrix as:

$$
R_{Z Z}=\frac{1}{n} \sum_{k=1}^{n} Z\left(t_{k}\right) Z^{*}\left(t_{k}\right)
$$

The eigen decomposition of the positive definite and hermetian sample covariance matrix $k_{z z}$ is given by

$$
\hat{R}_{\mathrm{ZZ}}=\sum_{i=1}^{2 m} \lambda_{i} e_{i} e_{i}^{*}=E_{s} \Lambda_{s} E_{s}^{*}+\sigma^{2} E_{n} E_{n}^{*}
$$


where $\Lambda_{s}$ is a diagonal matrix with $\lambda_{1} \geq \lambda_{2} \geq \ldots \geq \lambda_{d}>\lambda_{d+1}=\ldots=\lambda_{2 m}$

$E_{S}=\left[e_{1}, e_{2}, \ldots, e_{d}\right]$ is the matrix composed of the eigenvectors corresponding to the $d$ largest eigen values that define the signal subspace, and $E_{n}=\left[e_{d+1}, e_{d+2}, \ldots, e_{2 m}\right]$ is the matrix of the eigenvectors that span the complement orthogonal noisesubspace. Since the matrices $E_{S}=\left(\begin{array}{c}E_{0} \\ E_{1}\end{array}\right)$ and $\bar{A}=\left(\begin{array}{l}A \\ A \Phi\end{array}\right)$ have the same range space, then the intent of the ESPRIT algorithm is to find a nonsingular matrix $T$ of rank $d$ such that

$$
\left(\begin{array}{c}
E_{0} \\
E_{1}
\end{array}\right)=\left(\begin{array}{l}
A \\
A \Phi
\end{array}\right) T
$$

By eliminating $A$ in (10), the following expression is obtained:

$$
E_{1}=E_{0} T^{-1} \Phi T=E_{0} \Psi
$$

where $\Psi=T^{-1} \Phi T$. In general, there is no matrix $\Psi$ that satisfies (11) exactly because $E_{0}$ and $E_{1}$ are equally noisy and their estimates do not span the same column subspace.

The conventional ESPRIT estimates $\Psi$ using the Least Square (LS) criterion, and thus mayyield in overall inferior results as the LS solution is known to be biased [11]. To circumvent the conventional ESPRIT algorithm drawback to some extent, the Total Least Square (TLS)-ESPRIT [11] is used instead to provide asymptotically unbiased and efficient estimates of the DOAs. The TLS estimates have been shown to be strongly consistent (convergeswith probability one to the true values). Following [11], a total least square (TLS) estimates of $\Psi$, given $E_{0}$ and $E_{1}$, is provided by

$$
\Psi_{T L S}=-E_{12} E_{22}^{-1}
$$

respectively, where $E_{12}$ and $E_{22}$ are implicitly defined by the eigen decomposition of

$$
E_{x y}^{*} E_{x y} \stackrel{d e f}{=}\left[\begin{array}{c}
E_{0}^{*} \\
E_{1}^{*}
\end{array}\right]\left[E_{0} \mid E_{1}\right]=\left[\begin{array}{ll}
E_{11} & E_{12} \\
E_{21} & E_{22}
\end{array}\right] L\left[\begin{array}{ll}
E_{11}^{*} & E_{12}^{*} \\
E_{21}^{*} & E_{22}^{*}
\end{array}\right]
$$

where $L=\operatorname{diag}\left(l_{1}, l_{2}, \ldots, l_{d}\right), l_{1}>l_{2}>\ldots>l_{d}$. 
In practical situations where only a finite number of noisy measurements are available, we have only an estimates of $\Psi_{T L S}$ of $\Psi$. In this case, the eigenvalues of $\Psi_{T L S}$, denoted by $\hat{\phi}_{i}, i=1, \ldots, d$, give estimates of the DOAs as:

$$
\hat{\theta}_{i}=\sin ^{-1}\left(\lambda \mu_{i} / 2 \pi D\right), i=1, \ldots, d \text {. }
$$

where $)_{i}=\arg \left(\stackrel{\prime}{\phi}_{i}\right)$.

\subsection{ESPRIT Performance}

As mentioned earlier, the TLS-ESPRIT algorithm[11] leads to unbiased estimates phase shifts under the assumption that a very large number of data snapshots are available, a condition difficult to satisfy in practical situations. When the collected data snapshots are limited, the estimates do not always approach the asymptotic ones, and the realizable results vary as function of the estimation error variance of the phase shifts.

In general, if we consider that the estimated phase shifts $\mu$ of $\mu$ are determined with an estimation error $\Delta \mu$, and with the assumption that $\Delta \mu$ is a Gaussian independent process with zero mean and with variance $E\left(\Delta \mu^{2}\right)=\sigma_{\mu}^{2}$, then from (1), the estimates of the DOAs $\dot{\theta}$ of $\theta$ are estimated with an error of

$$
\Delta \theta=\frac{\lambda(\Delta \mu)}{2 \pi D \cos \theta}
$$

Thus, $\Delta \theta$ is also Gaussian with zero mean and variance

$$
E\left(\Delta \theta^{2}\right)=\frac{\lambda^{2} \sigma_{\mu}^{2}}{4 \pi^{2} D^{2} \cos ^{2} \theta}
$$

One can clearly see that, as the absolute value of incidence angle $\theta$ increases, the variance of the error $\Delta \theta$ will also increase. For instance, consider the case of only one source impinging on the array with $\theta_{1}$ corresponding to a phase shift $\mu_{1}$. If this same source is impinging on the array with $\theta_{2}$, it will correspond to a phase shift $\mu_{2}$. With the assumption that $\left|\theta_{2}\right|>\left|\theta_{1}\right|$, and since $\sigma_{u_{1}}=\sigma_{\mu_{2}}=\sigma_{u}$, the forms of the Gaussian probability density functions of the estimated phase shifts with means $\mu_{1}$ and $\mu_{2}$ are shown in Figure 2. Likewise the Gaussian probability density functions of the corresponding estimated DOAs with mean $\theta_{1}$, and $\theta_{2}$, are shown in Figure 3 . If we choose the intervals $\mu_{1} \pm z_{\beta / 2} \sigma_{\mu}$ and $\mu_{2} \pm z_{\beta / 2} \sigma_{\mu}$,where $z_{\beta / 2}$ is the $z$ value that locates an area of $(1-\beta / 2)$ in the upper tail of a normal distribution, then we are certain that these intervals will contain estimates of $\mu_{1}$ and $\mu_{2}$ with a probability equal to $(1-\beta)$ or a confidence level $\mathfrak{I}=(1-\beta) 100 \%$. However, we point out that while maintaining a constant confidence interval 
for the estimates of the phase shifts, the confidence interval of the estimates of the DOAs will increase with the angle of incidence. This is depicted in Figure 3, where the confidence interval for the estimates of $\theta_{2}$ is clearly wider than the confidence of the estimates of $\theta_{1}$ since the error of the estimates of $\theta_{2}$ has larger sample variance than the error of the estimates of $\theta_{1}$.

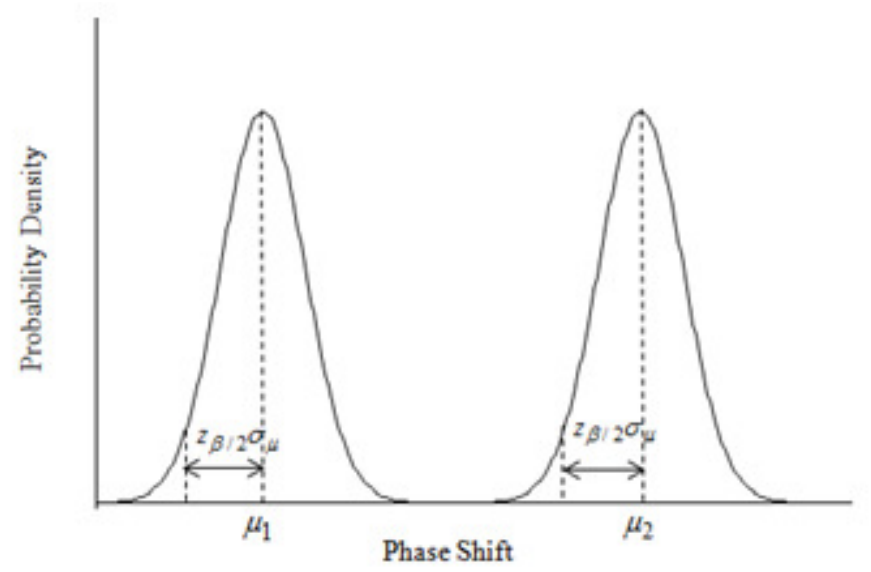

Figure2. Probability Density Functions of the Phase Shifts

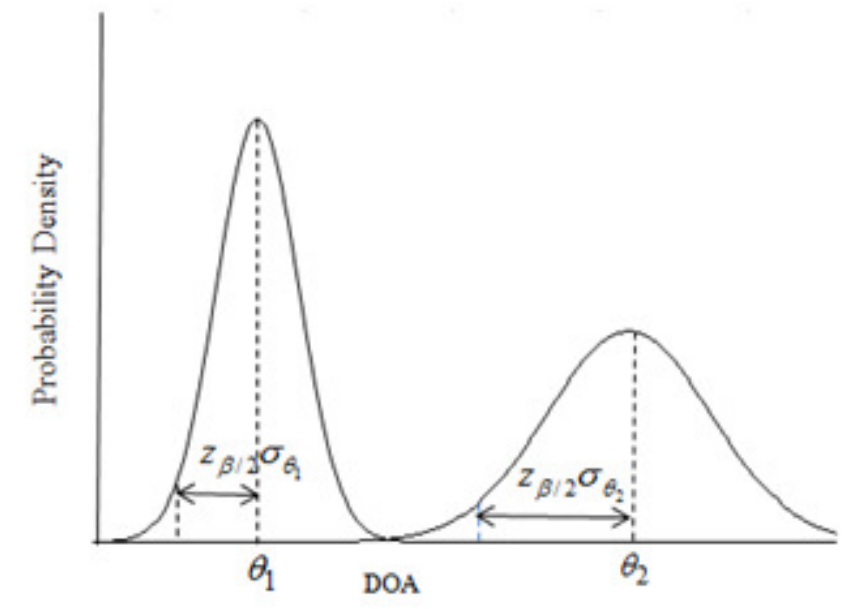

Figure 3. Probability Density Functions for the DOAs

It is obvious that (16) cannot be made arbitrary small, especially for angles of incidence alongside the ULA, without putting a constraint on $\sigma_{\mu}$. Maintaining a good accuracy of the estimated DOA simplies having a very small $\sigma_{\mu}$. This means a higher SNR and/or an increase of the number of snapshots $n$. For instance, this is clearly shown for the one source case [3], where the phase shift estimation error, the $S N R$, and the number nof snapshots are related by

$$
\sigma_{\mu}^{2}=\frac{1}{S N R}\left(\frac{1}{m^{2} n}\right) .
$$


For more than one source, the relationship between $\sigma_{\mu}$ and the $S N R$ and number of snapshots $n$ is more complicated [7]. Nevertheless due to the non-linear relationship between the phase shift and the DOA given by (1), the basic trend is that higher $S N R$ and/or larger $n$ are required to obtain more accurate DOA estimates or to distinguish between two or more close sources as the absolute of their angles of incidence increases. That is, ESPRIT is limited by its ability to resolve two closely spaced sources when their angles of incidence are along-side the ULA. In fact, for low $S N R$ and limited number of snapshots, ESPRIT algorithm will fail in this case to determine that there are actually two sources present. It will indicate that there is only one source present.

\subsection{Cyclic Ambiguities}

Consider the relation, given by (1), where we have tacitly assumed that $D=f(\lambda)$ holds perfectly. In practice however, due to measurement errors, this holds only approximately. Let $\Delta D$, represent the error on $D$, this will introduce an error on the phase shift as

$$
\Delta \mu=\frac{2 \pi \Delta D \sin \theta}{\lambda}
$$

Because the effective angular range of the ULA has a value $-90^{\circ} \leq \theta \leq 90^{\circ}$, the phase shift has a

value in the range $-\pi \leq \mu<\pi$. Since the inverse of the mapping $\mu \rightarrow e^{-j u}$ is aliased outside this range, then any error on the phase shift, given by (18), may introduce cyclic ambiguities in estimating the DOAs. For illustration purposes, suppose that $D=0.56 \lambda(>\lambda / 2)$ and a source impinging on the array with $\theta=85^{\circ}$, that correspond to a phase shift of $\mu=3.505$. This phase shift is cyclically equivalent to a phase shift of $\mu=-2.777$. ESPRIT will misinterpret this value and compute the estimated DOA, assuming $D=\lambda / 2$, to be $\theta=-62.16^{\circ}$ which is very different from the true DOA at $85^{\circ}$.

In short, ambiguous errors due to misinterpretation of cyclically ambiguous phase shift occur in ESPRIT if

$$
\Delta \mu>\pi-|\mu|
$$

Similarly, for low $S N R$ and small number of snapshots, cyclic ambiguities may occur in estimating the DOAs for sources impinging on the array at an angle of incidence along-side the ULA. For $D=\lambda / 2$, consider the phase shift $\mu=\pi \sin \theta$, resulting for a $\theta$ close to $90^{\circ}$, as shown in Figure 4 .

It is noticeable that a small perturbation on the phase shift may lead to a phase shift $\hat{\mu}_{1}>\pi$. This phase shift is cyclically equivalent to the phase shift $\hat{\mu}$. Again, ESPRIT algorithm will misinterpret the estimated phase and computes the estimated DOA at a value that is a very different from the true DOA. 


\section{PROPOSED METHOD}

In this work, a method is proposed to address the ambiguity, resolution and accuracy problems of the DOA estimation.

\subsection{DOA Estimation}

From Figure 5, showing the phase Shift as function of the Angle of Arrival for $D=\lambda / 2$, one can notice that if the DOA is kept within the range $-30^{\circ} \leq \theta \leq 30^{\circ}$, then the phase shift $\mu$ can be approximated as

$$
\mu=\frac{2 \pi D \sin \theta}{\lambda} \approx \frac{2 \pi^{2} D \theta}{180 \lambda}
$$

where $\theta$ is expressed in degrees. This implies that $\Delta \mu \approx \frac{2 \pi^{2} D}{180 \lambda} \Delta \theta$, and thus approximately the same $S N R$ and/or the same number of snapshots are needed to maintain the same accuracy of the DOAs.

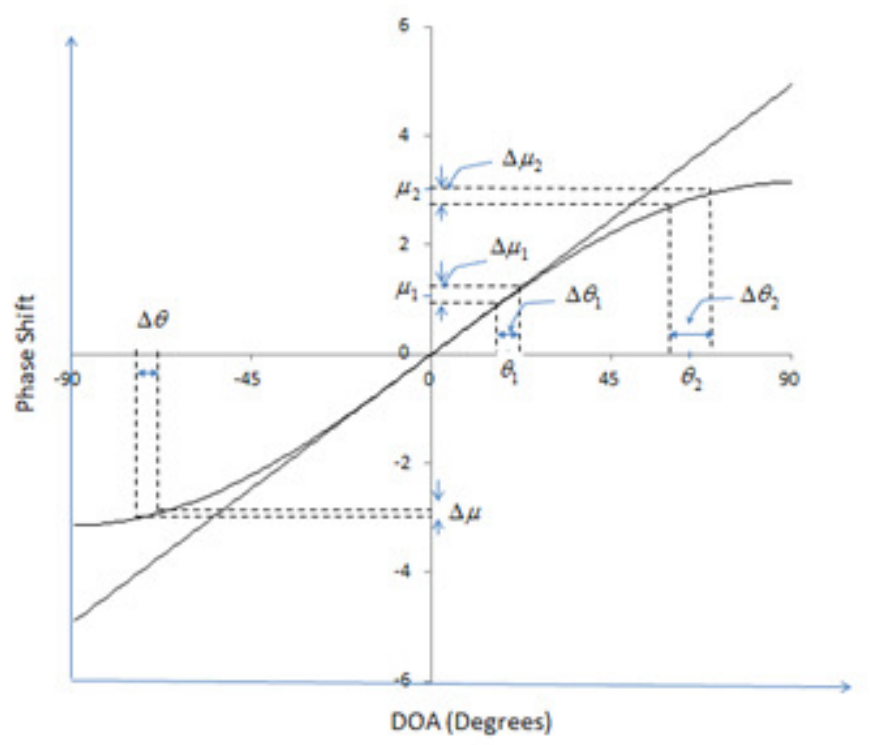

Figure 5.Phase Shift as function of the Angle of Arrival for $D=\lambda / 2$

Thus, the main idea behind the new method is to map any estimate $\dot{\theta}$ of the DOA that iswithin the range of $-90^{\circ} \leq \hat{\theta} \leq-30^{\circ}$ or $30^{\circ} \leq \hat{\theta} \leq 90^{\circ}$ into the range of $-30^{\circ} \leq \dot{\theta} \leq 30^{\circ}$. This calls for the exploration of rotating the array in the elevation plane, as shown in Figure 6, and sampling the received signals at three different positions. 


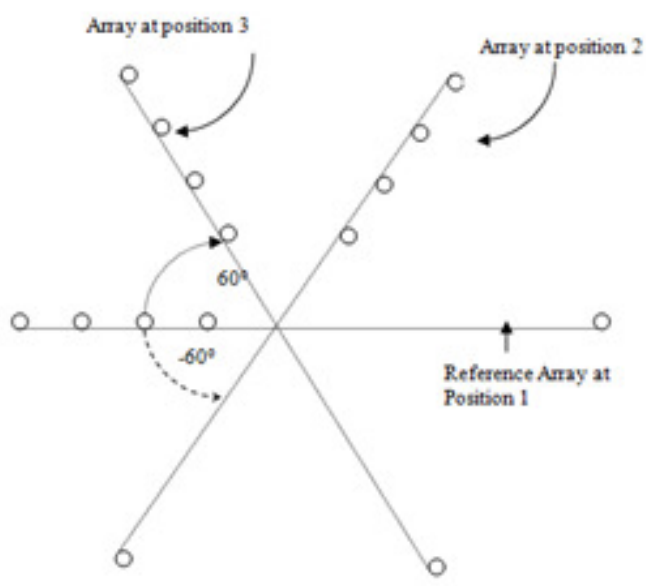

Figure 6. Array Rotation Positions

In this case, one expects to get three estimates of the DOAs at the different array positions in the range of $-90^{\circ} \leq \hat{\theta}^{(1)} \leq 90^{\circ},-30^{\circ} \leq \hat{\theta}^{(2)} \leq 150^{\circ}$, and $-150^{\circ} \leq \hat{\theta}^{(3)} \leq 30^{\circ}$, respectively. However because the estimated phase shifts outside the range of $-\pi \leq \mu<\pi$ are aliased, any angle $\hat{\theta}^{(2)}$ in the range $90^{\circ} \leq \hat{\theta}^{(2)} \leq 150^{\circ}$ will appear in the range $30^{\circ} \leq \hat{\theta}^{(2)} \leq 90^{\circ}$, and any angle $\dot{\theta}^{(3)}$ in the range $-150^{\circ} \leq \hat{\theta}^{(3)} \leq-30^{\circ}$ will appear in the range $-90^{\circ} \leq \hat{\theta}^{(3)} \leq-30^{\circ}$, as depicted in Figure 7.Thus, by considering only the estimates of DOAs in the range of $-30^{\circ} \leq \hat{\theta} \leq 30^{\circ}$ at each array position, we have the following cases:

a) If $\dot{\theta}_{i}^{(1)}$ is within the range $-30^{\circ} \leq \hat{\theta}^{(1)} \leq 30^{\circ}$, the actual DOA estimate is $\dot{\theta}_{i}=\hat{\theta}_{i}^{(1)}$.

b) If $\hat{\theta}_{i}^{(2)}$ is within $-30^{\circ} \leq \hat{\theta}^{(2)} \leq 30^{\circ}$, the actual DOA estimate is $\hat{\theta}_{i}=\dot{\theta}_{i}^{(2)}-60^{\circ}$.

c) If $\hat{\theta}_{i}^{(3)}$ is within $-30^{\circ} \leq \hat{\theta}^{(3)} \leq 30^{\circ}$, the actual DOA estimate is $\hat{\theta}_{i}=\hat{\theta}_{i}^{(3)}+60^{o}$.

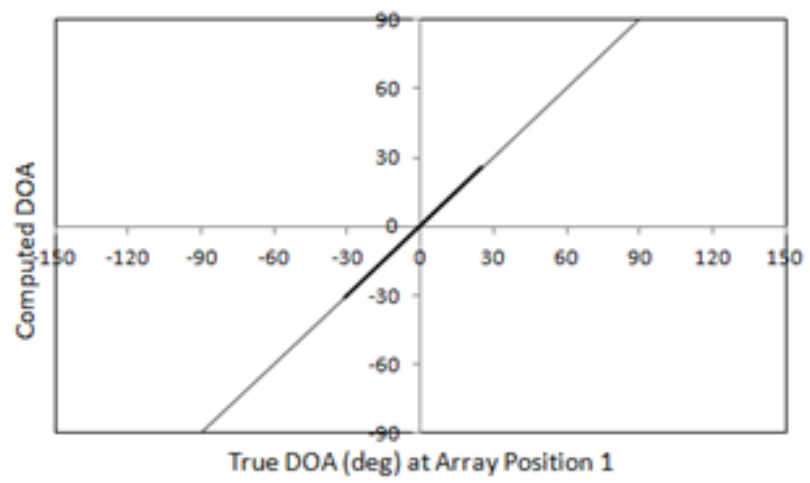

(a) 


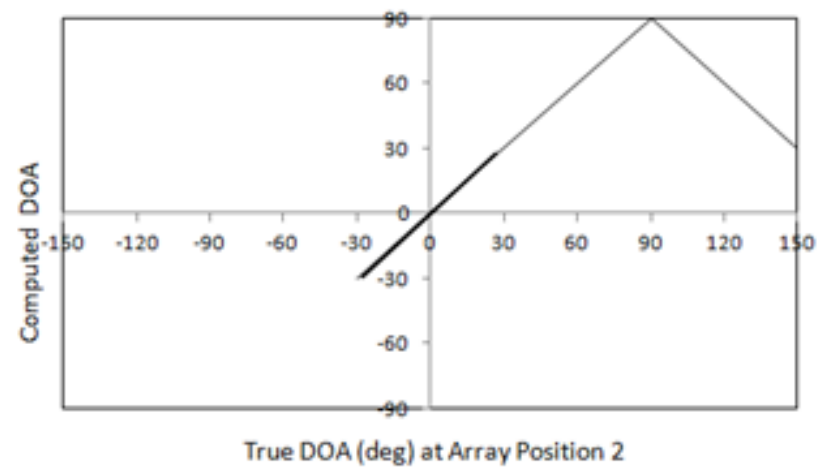

(b)

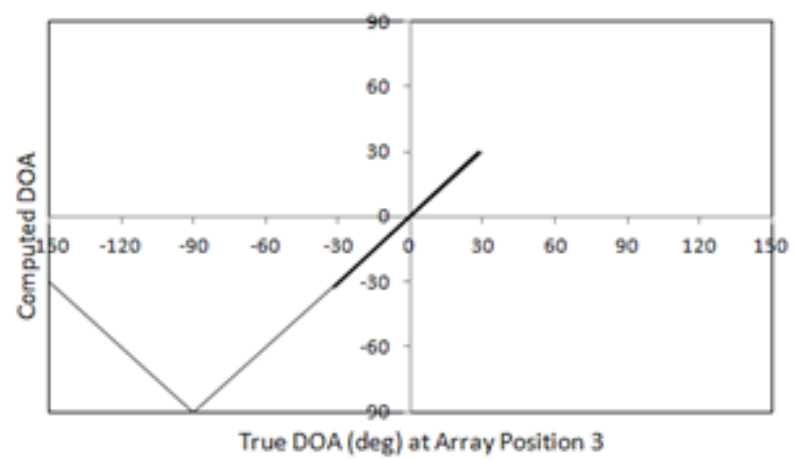

(c)

Figure 7. Computed DOAs as Function of the True DOAs at the Different Array Positions

To be more specific the estimation of the DOAs is performed as follows:

Step 1: Using (8), get estimates of the covariance matrices $k_{z z_{1}}, R_{z z_{2}}$, and $k_{z z_{3}}$, obtained from the data collected at array positions1, 2and3, respectively.

Step 2: Compute the generalized eigen decompositions of $\left\{R_{Z Z_{1}}, I_{n}\right\},\left\{R_{Z Z_{2}}, I_{n}\right\}$, and $\left\{k_{\mathrm{ZZ}_{3}}, I_{n}\right\}$, where $I_{n}$ is the identity matrix.

Step 3: Using Akaike's information criterion (AIC) or the minimum description length (MDL)[12], estimate the number of sources $d_{1}, d_{2}$ and $d_{3}$ at each array position. Note that ESPRIT may be limited by its ability to resolve two closely spaced sources when their angles of incidence are along-side the ULA, the estimation of the number of sources may vary at the different array positions.

Step 4: Use(9)-(11) to obtain the signal spaces estimates, composed of the eigenvectors corresponding to the $d_{1}, d_{2}$, and $d_{3}$ largest eigenvalues of $k_{Z Z_{1}}, k_{z z_{2}}$, and $k_{z z_{3}}$, respectively. 
Step 5: Using (12), obtain the corresponding three mapping matrices $\Psi_{1 T L S}, \Psi_{2 T L S}$ and $\Psi_{3 T L S}$

Step 6: Estimate the DOA's $\dot{\theta}_{i}^{(j)}=\sin ^{-1}\left(\mu_{i}^{(j)} \lambda / 2 \pi D\right)$, where $\mu_{i}^{(j)} ; i=1, \ldots d_{j} ; j=1,2,3$. are the phases shifts obtained from the eigen decomposition of $\Psi_{1 T L S}, \Psi_{2 T L S}$ and $\Psi_{3 T L S}$, respectively.

Step 7: By considering only the estimates of DOAs in the range of $-30^{\circ} \leq \hat{\theta} \leq 30^{\circ}$, classify them according the three cases specified above.

The above algorithm also resolves ambiguities in estimating the DOAs.

For $D=\lambda / 2$, if we denote by $\theta^{(1)}, \theta^{(2)}$, and $\theta^{(3)}$ the angle of incidence at the different positions of a source impinging on the reference array with $\theta$, and by $\mu^{(1)}, \mu^{(2)}$, and $\mu^{(3)}$ their corresponding phases shifts, then we will have the following three cases detailed in Table.1

Table 1. DOAs and Corresponding Phase Shift at the Different Array Positions

\begin{tabular}{|l|l|l|}
\hline & DOA & Phase Shift \\
\hline Case 1: & $-30^{\circ} \leq \theta^{(2)} \leq 30^{\circ}$ & $-\pi / 2 \leq \mu^{(2)} \leq \pi / 2$ \\
$-90^{\circ} \leq \theta \leq-30^{\circ}$ & $-90^{\circ} \leq \theta^{(1)} \leq-30^{\circ}$ & $-\pi \leq \mu^{(1)} \leq-\pi / 2$ \\
& $-90^{\circ} \leq \theta^{(3)} \leq-30^{\circ}$ & $-\pi \leq \mu^{(3)} \leq-\pi / 2$ \\
\hline Case 2: & $30^{\circ} \leq \theta^{(2)} \leq 90^{\circ}$ & $\pi / 2 \leq \mu^{(2)} \leq \pi$ \\
$-30 \leq \theta \leq 30$ & $-30^{\circ} \leq \theta^{(1)} \leq 30^{\circ}$ & $-\pi / 2 \leq \mu^{(1)} \leq \pi / 2$ \\
& $-90^{\circ} \leq \theta^{(3)} \leq-30^{\circ}$ & $-\pi \leq \mu^{(3)} \leq-\pi / 2$ \\
\hline Case 3: & $30^{\circ} \leq \theta^{(2)} \leq 90^{\circ}$ & $\pi / 2 \leq \mu^{(2)} \leq \pi$ \\
$30 \leq \theta \leq 90$ & $30^{\circ} \leq \theta^{(1)} \leq 90^{\circ}$ & $\pi / 2 \leq \mu^{(1)} \leq \pi$ \\
& $-30^{\circ} \leq \theta^{(3)} \leq 30^{\circ}$ & $-\pi / 2 \leq \mu^{(3)} \leq \pi / 2$ \\
\hline
\end{tabular}

By considering the phase shifts in the complex plane as shown in Figure 8 for the different cases, only the phase shifts that are in quadrant 1 or quadrant 4 should be considered to estimate the DOAs. All others should be rejected. However, one can notice that ambiguities only occur if the estimated phase shifts that are intended to be in quadrant 2 or quadrant 3 are misclassified to be in quadrant 1 or quadrant 4. However, for this to happen, it will require a perturbation on the phase shift, given by:

$$
\Delta \mu>|\mu|-\pi / 2
$$


For illustration purposes, suppose that $\theta=85^{\circ}$. Using the conventional case, this corresponds to a phase of $\mu=3.1296$. From (19), any estimate of the phase shift that has a deviation of $\Delta \mu>0.012$ from the true (noiseless) phase shift will introduce ambiguities.

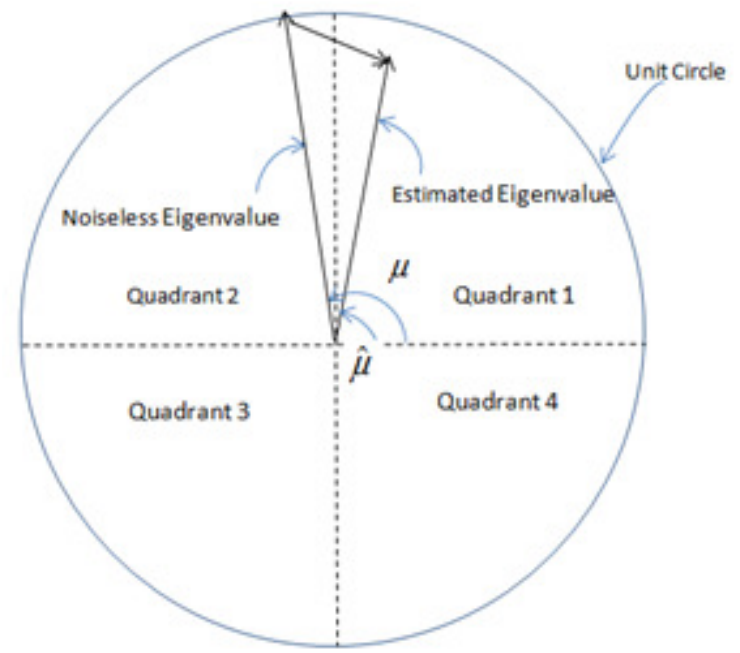

Figure 8. Error Effect on the Eigen value Estimation when the three arrays are used.

Now, using the new algorithm, a $\theta=85^{\circ}$ will correspond to $\theta^{(1)}=85^{\circ}\left(\mu^{(1)}=3.1296\right)$, $\theta^{(2)}=145^{\circ}\left(\mu^{(2)}=1.8019\right)$, and $\theta^{(3)}=25^{\circ}\left(\mu^{(3)}=1.3277\right)$. Note that $\mu^{(2)}$ also corresponds to the aliased value $\theta^{\prime(2)}\left(=35^{\circ}\right)$ of $\theta^{(2)}$.

It is clear that estimates of $\theta^{(3)}$ that are in quadrant 1 , offset by $60^{\circ}$, will be considered to be the estimates of the actual DOAs. However, since $\mu^{(2)}$ is close to $\pi / 2$, ambiguities may still occurif any of its estimates that are intended to be in quadrant 2are misclassified to be in quadrant 1 . In this case, the new ESPRIT algorithm will compute the estimated DOA with an offset of $-60^{\circ}$ instead of an offset of $60^{\circ}$, which leads to a value that is a very different from the true DOA.But for this to occur, and using (22), it will require a deviation of $\Delta \mu>0.231$ from the noiseless phase shift, as compared to $\Delta \mu>0.012$ using the conventional algorithm. To completethis discussion, the performances of the two cases are compared. The criterion used for this comparison is the probability $\mathrm{Pa}$ that ambiguities will occur. For the conventional case,and using (19), the probability of ambiguities can be easily shown to be given by the complementary error function

$$
P_{a}^{(1)}=\frac{1}{2} \operatorname{erfc}\left(\frac{\pi-|\mu|}{2 \sigma_{u}}\right)
$$

Now considering the new method, the probability of ambiguities is given by: 


$$
P_{a}^{(2)}=\frac{1}{2} \operatorname{erfc}\left(\frac{\left|\mu^{(1)}\right|-\pi / 2}{2 \sigma_{u}}\right)+\frac{1}{2} \operatorname{erfc}\left(\frac{\left|\mu^{(2)}\right|-\pi / 2}{2 \sigma_{u}}\right)
$$

Using the same example for one source impinging on the array with $\theta=85^{\circ}$ and $D=\lambda / 2, P_{a}^{(1)}$ and $P_{a}^{(2)}$ are plotted in Figure 9 for various values of the $S N R$, where $\sigma_{u}$ is computed from (17)with $m=8$ and $n=64$. The plot indicates that using the new algorithm eliminates ambiguities in this case even for low $S N R$.

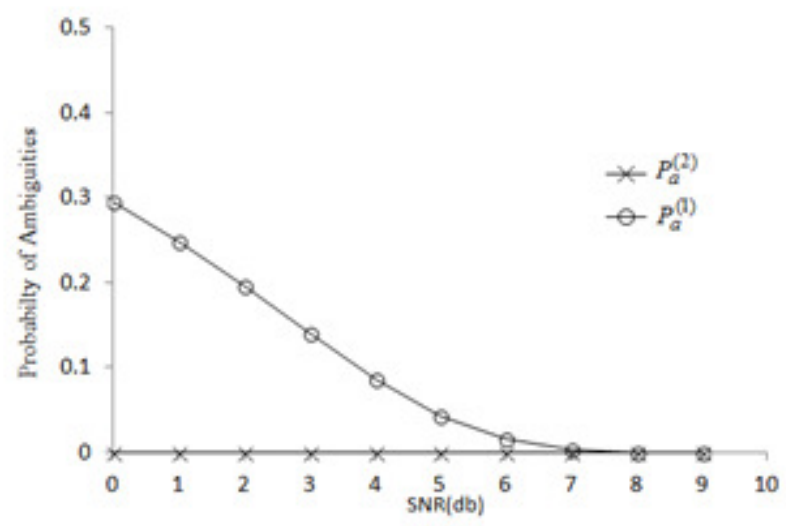

Figure 9. Probability of ambiguities

\section{SimUlation RESULTS}

In order to demonstrate the performance of the proposed method, the following simulations were performed. First to show the ambiguities in estimating the DOAs, $n(=64)$ snapshots were generated at the different $2 m$ sensors with a $S N R=O d B$ for one source impinging on the reference array with DOA of $85^{\circ}$. The result is brought out by presenting in Figure 10, estimates of the phase shifts resulting from 10 experiments. One observation may be made at this point. When using the conventional method, out of the 10 estimates of the phase shifts, 3 of the estimates are in quadrant 3 , resulting in an ambiguous estimation of the DOAs.

However, when considering the estimates derived from the data collected at the different positions of the array, one can notice that all the estimates of $\mu^{(3)}$ are included in quadrant 1.

These estimates will be used to estimate the actual DOAs. One also can notice that no ambiguities are occurring as all the estimates of phase shifts $\mu^{(1)}$ and $\mu^{(2)}$ are either within quadrant 2 , orquadrant 3 . This is an encouraging result in when compared to the result wherethe conventional algorithm is used. 


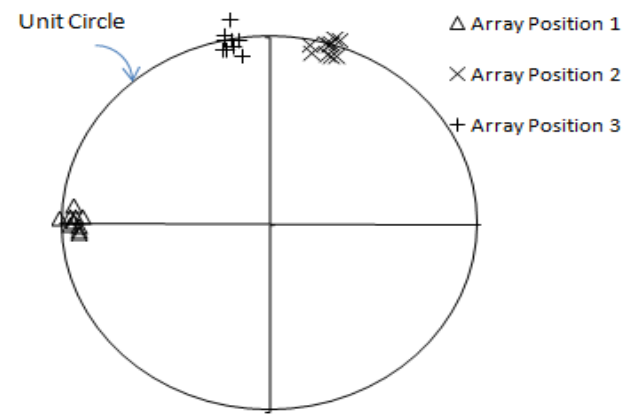

Figure 10. Estimated phase shifts obtained from data collected at the three different arrays positions

To show that for a low $S N R$ and limited number of snapshots, ESPRIT algorithm fails to distinguish between two or more close sources when the angles of their incidence is along-side the ULA, two sources with DOAs of $70^{\circ}$ and $75^{\circ}$ were considered to be impinging on the array. We notice that when the conventional ESPRIT algorithm is used, it fails to distinguish between the two sources for low SNR, as shown in Figure 11(a). However, the new algorithm always distinguishes between the two sources even for low SNR as shown in Figure 11(b).

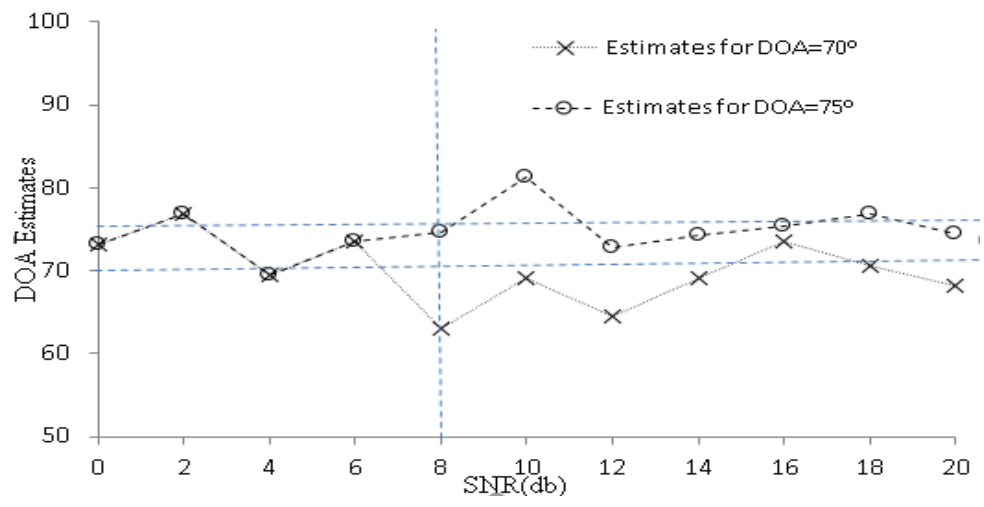

(a)

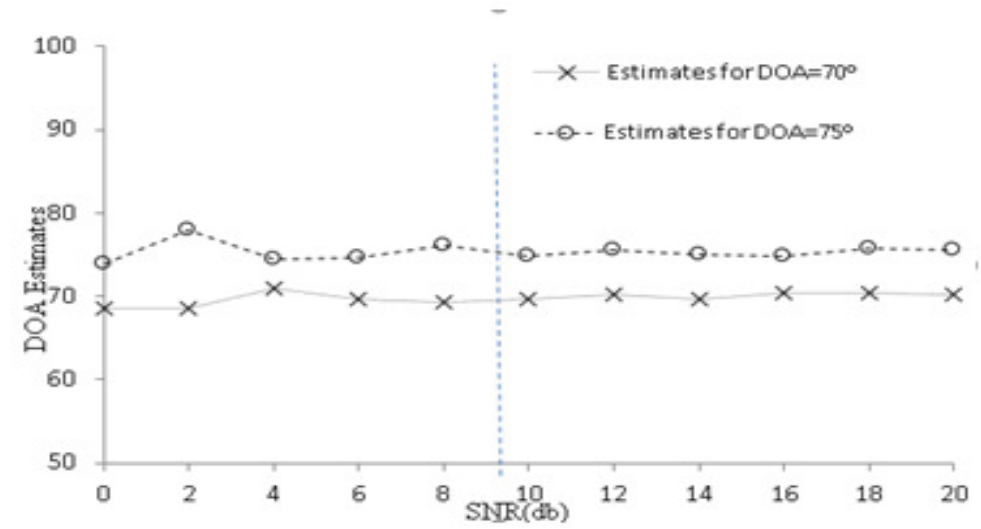

(b)

Figure 11. DOA Estimates Using the Conventional and the New Algorithms 
Now, in order to demonstrate further advantages of the performance of the proposed method, the same two sources with DOAs of $70^{\circ}$ and $75^{\circ}$ were considered to be impinging on the array. $n(=1000)$ snapshots were collected at each array position. The performance of new approach is brought out by examining the results illustrated in Figure 12, and Figure 13, where the bias and standard deviation are plotted respectively. The estimated DOAs were averaged over 100 experiments. To make the figure less crowded, only the results of DOA of $70^{\circ}$ are shown. By rejecting any estimate outside the range of $-30^{\circ} \leq \hat{\theta} \leq 30^{\circ}$, only the estimates of $\theta^{(2)}$ were considered, and these estimates were offset by $60^{\circ}$ to get the actual estimates. To show the gain in accuracy, the estimates were compared with the ones obtained in the conventional way which corresponds in this case to the estimates of $\theta^{(1)}$. As expected, the new algorithm performed far better, especially for low $S N R$. It should be noted that below a $S N R=8 d b$, the conventional ESPRIT failed to distinguish between the two sources or introduced ambiguities, and thus its estimates were excluded in this range

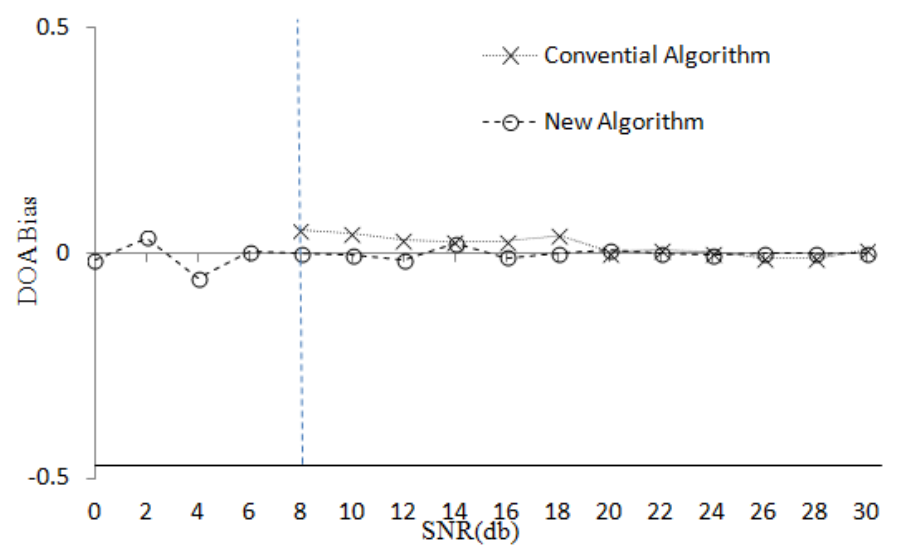

Figure 12. DOA Bias for the Conventional and the New Algorithms

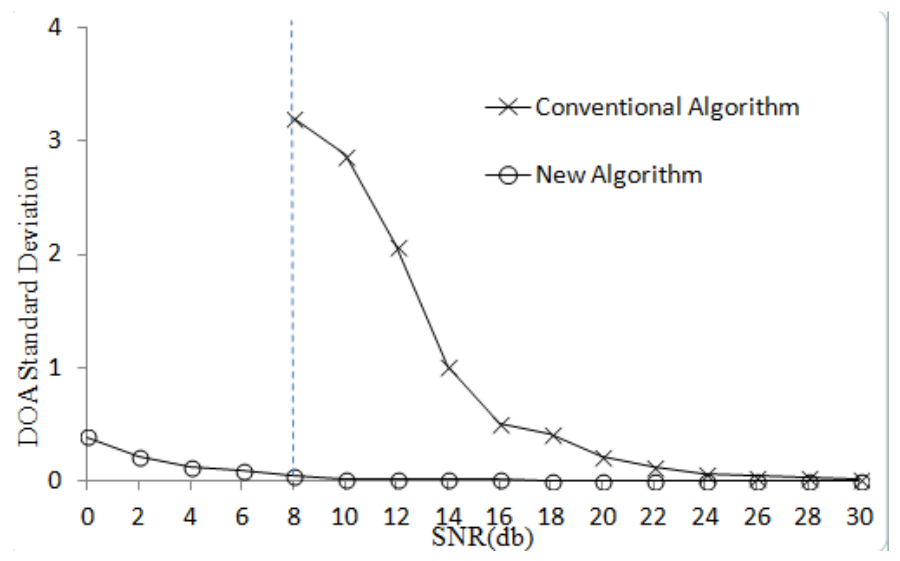

Figure 13. DOA Standard Deviation for the Conventional and the New Algorithms 


\section{CONCLUSION}

The primary goal of this study has been to develop a procedure to resolve the cyclic ambiguities of the DOA estimates, and increase their resolution and accuracy. Although the new approach calls for the exploration of rotating the array and sampling the received data of signals at multiple positions, this disadvantage is overcome by the gain in accuracy and resolution in the DOA estimation, and the considerable improvement in reducing the ambiguities that are more likely to occur when the angle of incidence is along-side the ULA, especially for low SNR and small number of snapshots. Note that one can first only use the reference array (conventional method) in estimating the DOAs. If any estimates are found to be in the range $-90^{\circ} \leq \hat{\theta} \leq-30^{\circ}$ or $30^{\circ} \leq \hat{\theta} \leq 90^{\circ}$, then these estimates are considered to be coarse estimates, and the array will be rotated by $60^{\circ}$ and $-60^{\circ}$ to obtain estimates at a finer scale.

\section{REFERENCES}

[1] E. Tuncer and B. Friedlander, (2009) Classical and Modern Direction-of-Arrival Estimation, Ed. Elsevier, USA.

[2] F. F. Gao and A. B. Gershman, (2005) "A generalized ESPRIT approach to direction-of-arrival estimation," IEEE Signal Processing Letters, vol. 12, no. 3, pp. 254-257.

[3] N. P. Waweru, D. B. O. Konditi, P. K. Langat, (2014) "Performance Analysis of MUSIC, RootMUSIC and ESPRIT DOA Estimation Algorithm," International Journal of Electrical, Computer, Energetic, Electronic and Communication Engineering, Vol. 8, No:1, pp. 209-216.

[4] M. H. Bhede, D. G. Ganage, S. A. Wagh, (2015) "Performance Analysis of MUSIC and Smooth MUSIC Algorithm for DOA Estimation," International Journal on Recent and Innovation Trends in Computing and Communication, Vol. 3, Issue. 7, pp. 4397-4402.

[5] C. L. Srinidh, S. A. Hariprasad, (2012) "Comparative Study on Performance Analysis ofHigh Resolution Direction of Arrival Estimation Algorithms," International Journal of Advanced Research in Computer Engineering \& Technology, Volume 1, Issue 4, June 2012, pp. 67-79.

[6] K.T. Wong, M. D. Zoltowski,(1998) "Direction finding with sparse rectangular dual-size spatial invariance array”, IEEE Trans. Aerosp. Electron. Syst., 34, (4), pp. 1320- 1327.

[7] A. N. Lemma, A. J. van der Veen, and E. F. Deprettere, (1999) “ Multiresolution ESPRIT Algorithm," IEEE Transactions on Signal Processing, Vol. 47, No. 6, June 1999, pp. 1722-1726.

[8] V. I. Vasylyshyn,(2005) "Unitary ESPRIT-based DOA estimation using sparse linear dual size spatial invariance array”. Proc. European Radar Conf., Paris, France, pp. 157- 160.

[9] Tan, C. M., et al, (2002) "Ambiguity in MUSIC and ESPRIT for direction of arrival estimation," Electronics Letters, vol.38, no. 24, pp. 1598- 1600.

[10] K. Yang, Z. Zhao, X. Zhu, and Q. H. Liu, (2013) "Resolving ambiguities in DOA estimation by optimizing the element orientations," in Proc. IEEE Antennas Propag. Soc. Int. Symp. (APSURSI), Jul. 2013, pp. 1326-1327. 
[11] R. Roy and T. Kailath, (1989) "ESPRIT-Estimation of Signal Parameters Via Rotational Invariance Techniques," IEEE Transactions on Acoustics, Speech and Signal Processing, Vol. 37. No. 7, July 1989, pp. 984-995.

[12] D. B. Williams, (1999) "Detection: Determining the number of sources," in Digital Signal Processing Handbook, V. K. Madisetti and D. B. Williams, Eds. Boca Raton, FL: CRC.

\section{AUTHORS}

Abdelhamid Djouadi received the B.S. degree in electrical engineering from the University of Sciences and Technologies, Oran, Algeria, in 1980, and the M.S. and Ph.D. degree in electrical engineering from The Ohio State University, Columbus, OH, USA, in 1983 and 1987, respectively. After a successful academic career, since 1997, he has been with Nokia (former Alcatel-Lucent Technologies) as a technical lead driving improvement in $4 \mathrm{G}$ and $3 \mathrm{G}$ products. His research interests include wireless telecommunication, pattern recognition, sensor array, and signal processing.

Nebojsa I. Jaksic received the Dipl. Ing. degree in electrical engineering from Belgrade University in 1984, the M.S. in electrical engineering in 1988, the M.S. in industrial engineering in 1992, and the Ph.D. in industrial engineering in 2000 from The Ohio State University, Columbus, OH, USA. He is currently Professor at Colorado State University-Pueblo. Dr. Jaksic has published over 60 papers and holds two patents. He is a registered Professional Engineer with the State of Colorado. Dr. Jaksic is a senior member of IEEE and SME, and a member of ASEE. His research interests include robotics, automation, and nanotechnology.
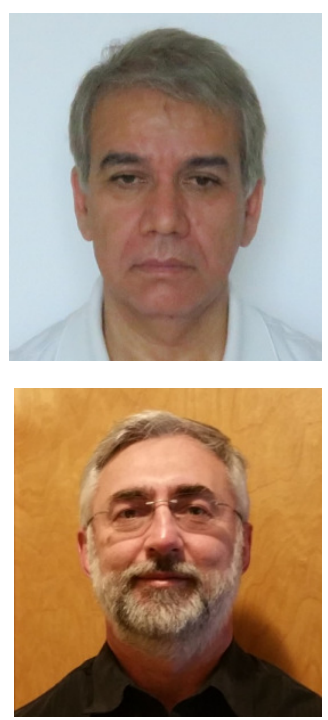